\title{
Method for Acquisition of Camera Document Images' Distorted Gradient
}

\author{
Yingying Wang, Huimin Lu,Jun Cai, Lifeng Zhang \\ Graduate school of Engineering, Kyushu Institute of Technology 1-1 Sensui-cho, Tobata, Kitakyushu, \\ Fukuoka, 804-8550 Japan, E-mail: 63645523@qq.com \\ Department of Information Engineering, Yangzhou University 88, South University Ave, Yangzhou, \\ Jiangsu, 225009, P. R. China,E-mail:26685993@qq.com \\ Graduate school of Engineering, Kyushu Institute of Technology 1-1 Sensui-cho, Tobata, Kitakyushu, \\ Fukuoka, 804-8550 Japan \\ E-mail: zhang@elcs.kyutech.ac.jp E-mail:serikawa@elcs.kyutech.ac.jp E-mail:bolandi@126.com
}

\begin{abstract}
Dewarping of camera document images has attracted a lot of interest over the last few years since warping not only reduces the document readability but also affects the accuracy of an OCR application. In this paper, approach for efficient getting distorted gradient of camera document images is presented. The gradient of text lines can be got by scanning black pixels' number in text. For avoiding only getting error gradient, the image is divided into 6 zones. In each zone, a gradient is found out. Then, the dewarping can be achieved by projective transformation. Experimental results on several document images demonstrate the robustness and effectiveness of the proposed technique.
\end{abstract}

Keywords: camera document image, gradient, pixels' number, dewarping, binary image

\section{Introduction}

Non-linear warping is often observed in document images, when captured by camera. Text in such cases is strongly distorted and influences the performance of further processing, since the contemporary OCR systems cannot handle distorted text.

Many different approaches have been proposed for document image dewarping [1-2]. These approaches can be classified into two broad categories based on [i] 3-D document shape reconstruction [3-8] and [ii] 2-D document image processing [9-20,26,27]. Our work is related to the second category. Previous approaches of the second category are described in the following:

$\operatorname{In}[26]$, the range rectified from a distortion picture may be specified. So this technique is that changing the outer skeleton of distorted image into rectangle one which has parallel before behind borders and parallel right left borders. However, by this technique, since there are restrictions that four sides before and behind the whole printed matter or the right and left of a print sheet are contained in a photography picture, if some printed matter is contained in the photography picture, it cannot be rectified.

[27] propose a method for document dewarping which has inclination of character strings using the characteristics of characters and interlinear spaces of the document. Long continuous branches, which define interlinear spaces of the document, are not parallel to each other in distorted picture. Find the horizontal vanishing point according the characteristics of characters, and vertical vanishing point by many vertical space bars between characters. The dewarping method is that make the straight lines which go to vanishing point parallel to each other. But if the interlinear spaces of the document are very narrow, the method does not work.

In this paper, we propose an approach for efficient getting the horizontal vanishing point of camera document images, which is directly applied to the 2D space, and can be used in document which has extremely narrow interlinear spaces. First step, divided the image into 6 zones. In each zone, rotate a short straight line on the point which it passes through; count the black pixels' number on the line. Second step, move the point and judge the distance between the point and characters according the variation of black pixels' number. Third step, when the point is close enough to the characters, rotate the line to make it parallel to characters. With the six gradients, calculate the horizontal vanishing point. Experimental results on several document images demonstrate the robustness and effectiveness of the proposed technique. The remainder of the paper is organized as follows. In section 2, the proposed 
technique is detailed while experimental results are discussed in Section 3. Finally, conclusions are drawn in Section 4.

\section{Proposed Method}

This paper is focused on horizontal distortion compensation when there are horizontal and vertical distortions in binary image. In this paper, we propose an approach for efficient getting the horizontal vanishing point of camera document images, which is directly applied to the 2D space, and can be used in document which has extremely narrow interlinear spaces. First step, divided the image into 6 zones. In each zone, rotate a short straight line on the point which it passes through; count the black pixels' number on the line. Second step, move the point and judge the distance between the point and characters according the variation of black pixels' number. Third step, when the point is close enough to the characters, rotate the line to make it parallel to characters. With the six gradients, calculate the horizontal vanishing point. Experimental results on several document images demonstrate the robustness and effectiveness of the proposed technique.

\subsection{Detecting black pixels}

As shown in Fig.1, the number of black pixels in the zone is detected by a scanning line which rotates on a point A. As shown in Fig. 2, the black pixels which are in a certain distance $\mathrm{d}$ from the line are taken into account and they are marked as $\circ$. Others are not and these pixels are marked as black points.

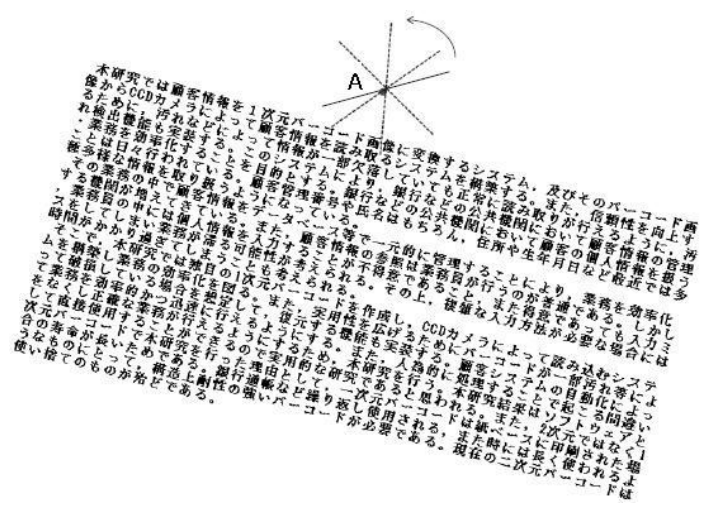

Fig.1 Rotate the line to detect pixels number.

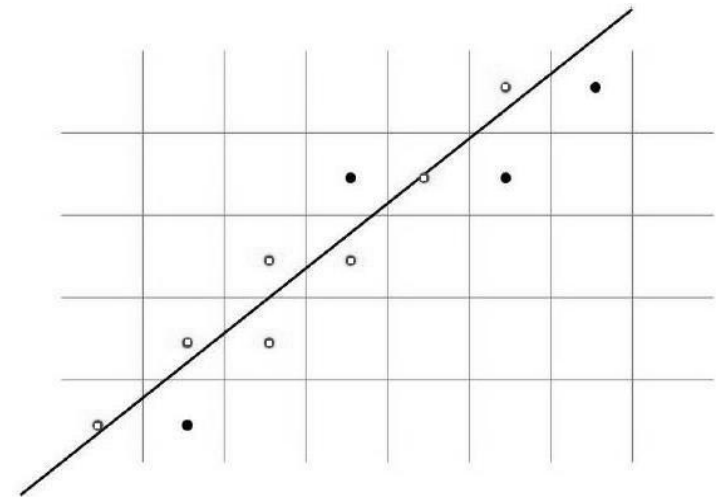

Fig. 2 Calculate the pixels' number.

\subsection{Getting the gradient of inclination character strings}

There are 2 steps in getting the gradient of warping document image. The 1st step is judge the position of point, to find out whether the point is close enough to text. If not, the 2nd step is approach it to the text. When the point is close enough to text, rotate the line to parallel to the text line. Then the gradient of line is the gradient of distorted text line.

\section{Step 1.Judge the Distance between Point and the Text}

At beginning, it should be judged that whether the point is close enough to text. There are all three situations between the point and text. Accordingly, there are three relationships between scanning line angles and detected pixels' number. As shown in Fig.3, if the point is far away from the text, there will be no pixel detected when the line rotates 180 degrees as shown in Fig.4.As shown in Fig.5, if the point is a little closer to the text, there will be some pixels detected in some scanned degrees as shown in Fig.6.As shown in Fig.7, if the point is in the text, there will be some pixels detected in all scanned degrees as shown in Fig.8. In this situation, if there is enough space between the strings, the horizontal vanishing point can be gotten. But if the space between the lines is very narrow, the point should be moved up to above the first line or down to below the last line. 


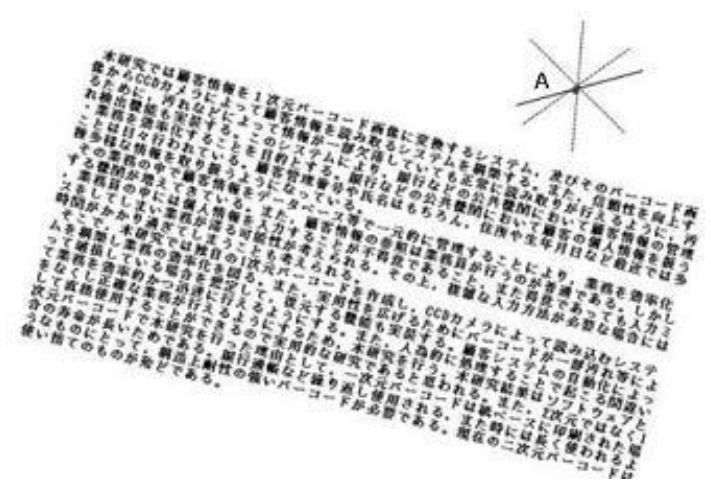

Fig.3 Point is far away from text.

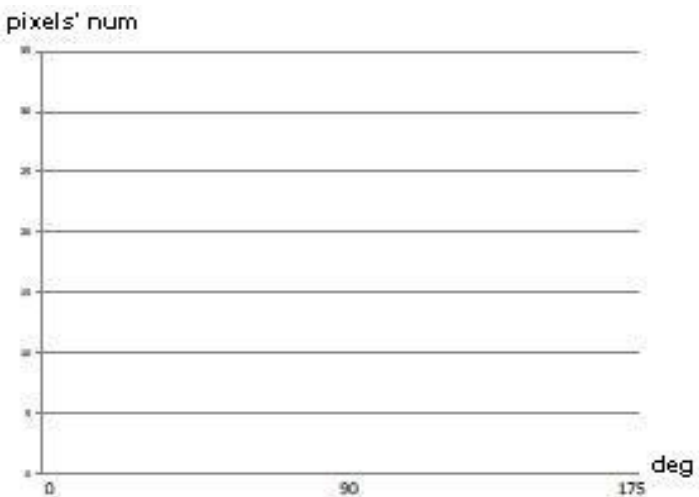

Fig.4 No pixels detected in 180 degree.

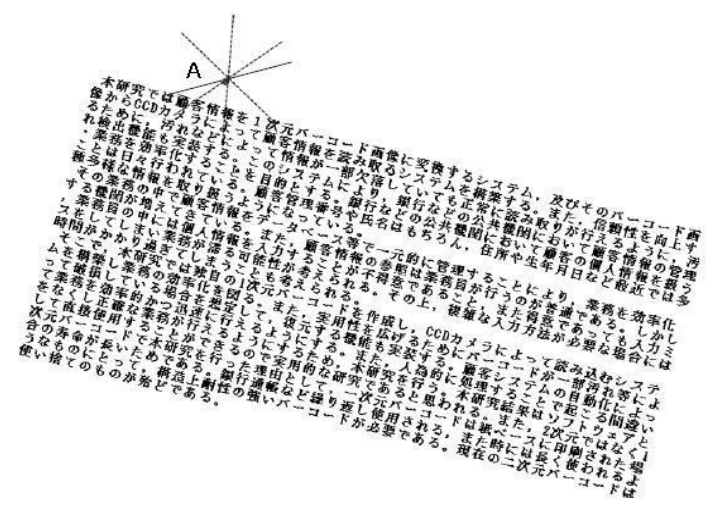

Fig. 5 Point is a little closer to text.

pixels' num

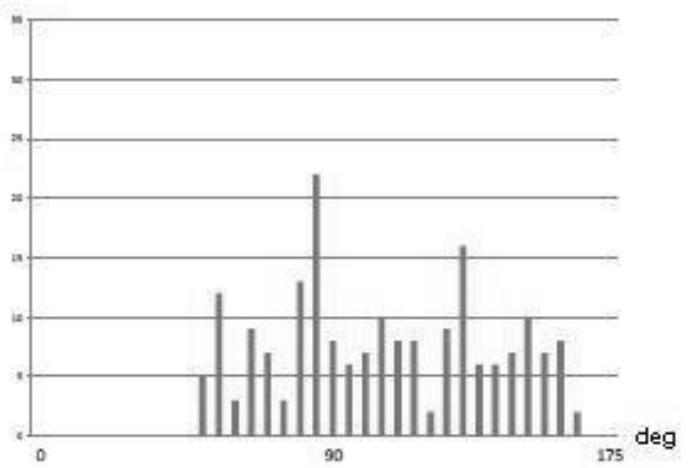

Fig.6 Pixels can be detected in some degrees.

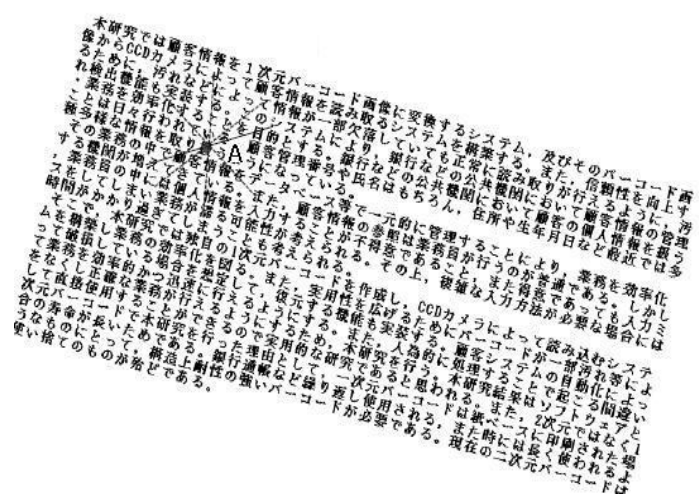

Fig.7 Point is in the text.

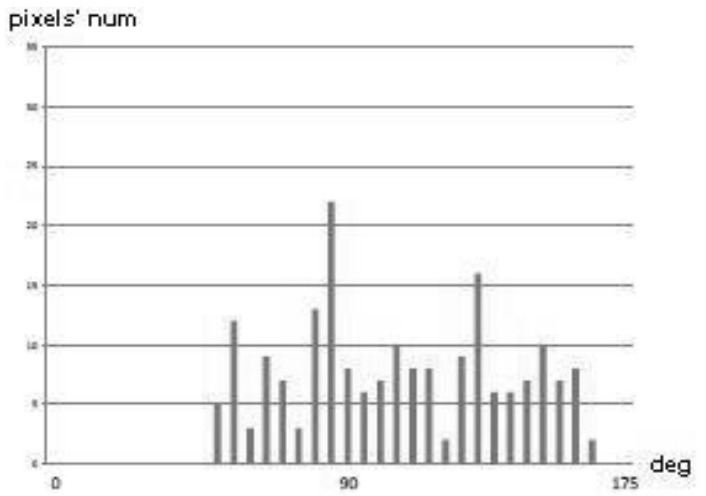

Fig.8 Pixels are detected in all degrees.

\section{Step 2. Approach the Point to strings}

Move the point, if it is moved away from the text, the degrees that pixels can be detected would decrease. Try to move the point to four directions and scan the pixels' number each time. Move to the direction where the degrees increase. A point which is close enough to text can be got out by repeating this operation as shown in Fig.11.The relationship between degrees and pixels' number is shown in Fig12.

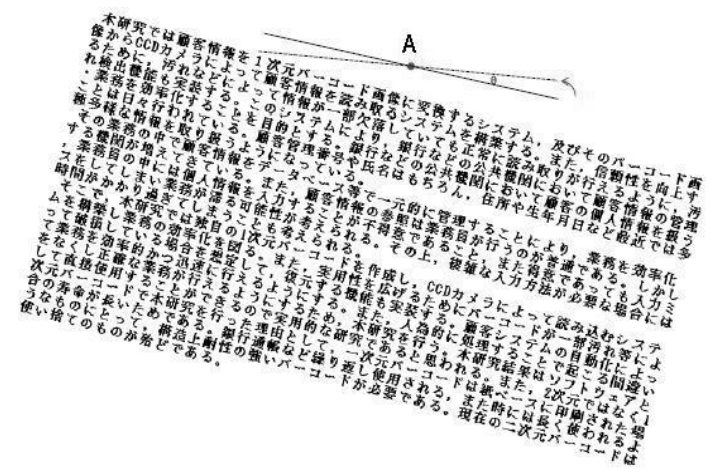

Fig.9 Point is moved away from text. 


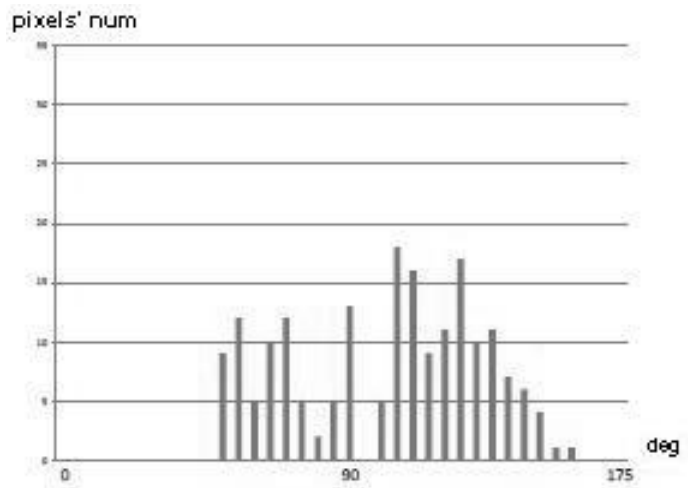

Fig.10 Pixels can be detected in less degrees.

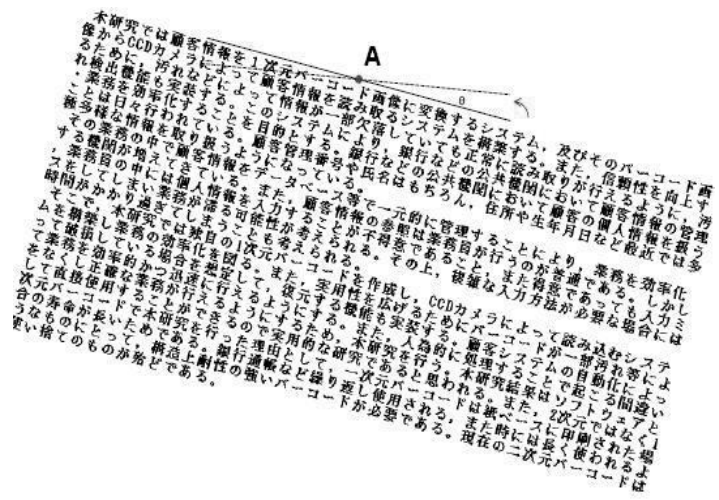

Fig.11Point is move right close to text.

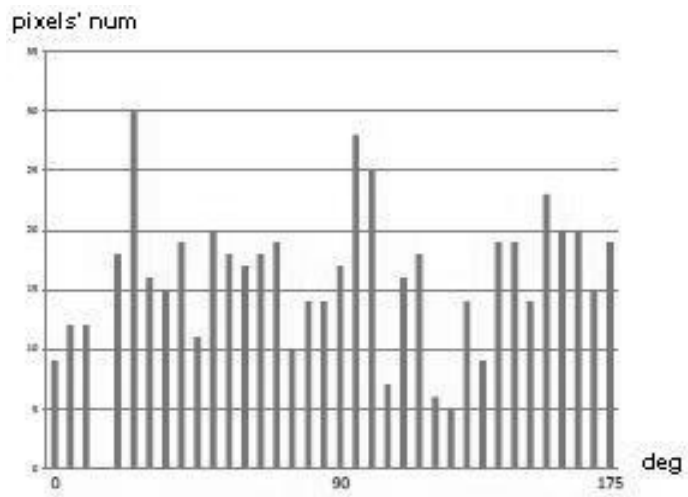

Fig.12 Pixels can be detected in 175degrees.

\section{Experiment results}

To verify the validity of the proposed method we take four set of images, SET-1, SET-2, SET-3,SET-4.SET-1 shows document with different character sizes. Two examples document images from the SET-1 are shown in Fig.13[a] and 13[b].SET-2 shows document with extremely narrow space between lines. An example document image from the SET-2 is shown in Fig.14.SET-3 shows document with English characters. An example document image from the SET-3 is shown in Fig.15. SET-4 shows document images with different incline gradients. Two example document images from the SET-4 are shown in Fig.16[a] and $16[\mathrm{~b}]$. The 4 SET experiments are successful.

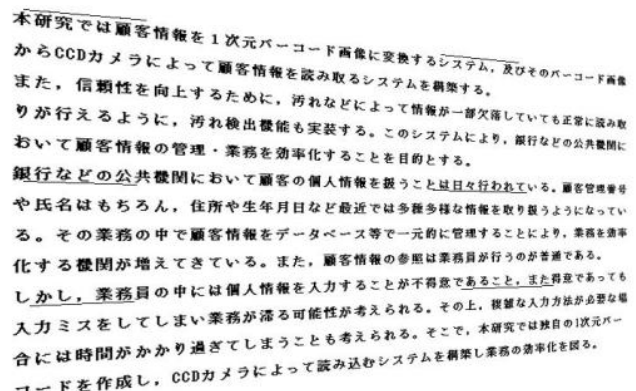

Fig.13 [a] The size of characters is $11 \mathrm{pt}$.

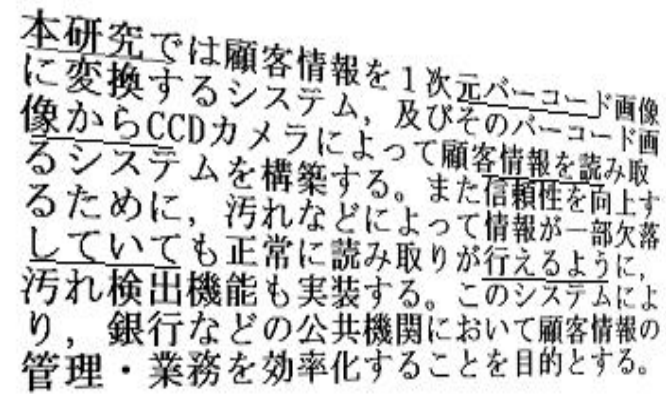

Fig. 13[b] The size of characters is $22 \mathrm{pt}$.

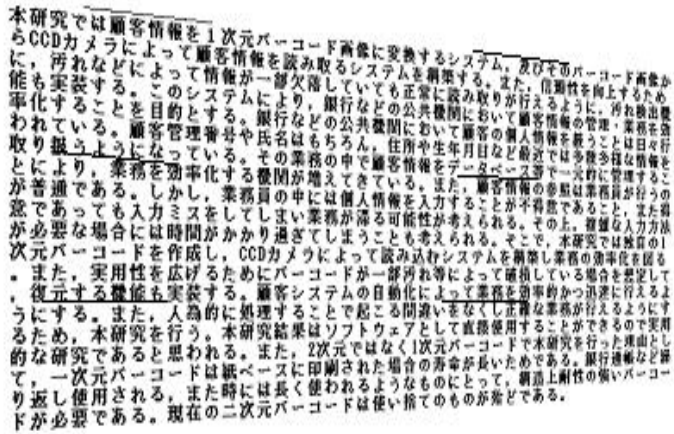

Fig.14 Interline spacing of text is very narrow. In the present study, system that converts information the system that reads the dimension bar code image and. from the bar code in information about customers Moreover, to read it normally even if information is missed to improve reliability due to dirt etc. partially. the dirt detecting function is mounted.

It aims to make management and the business of the It aims to make management afficiency in a public information about customers by this system. organization of the bank etc. by this syctem 
Fig.15 Document text is English.

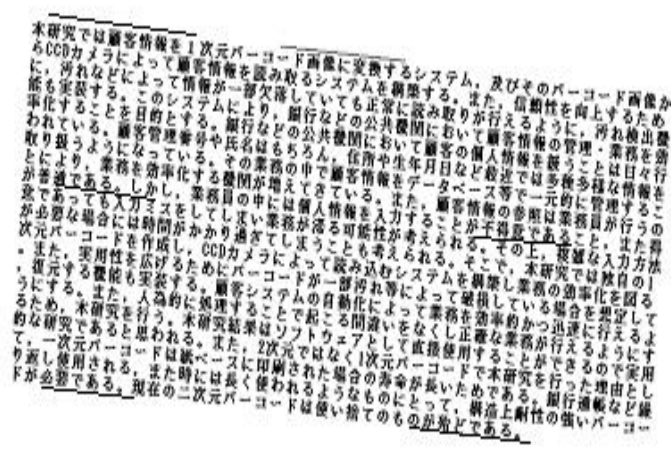

Fig. 16[a] Incline gradient of document is $5^{\circ}$.

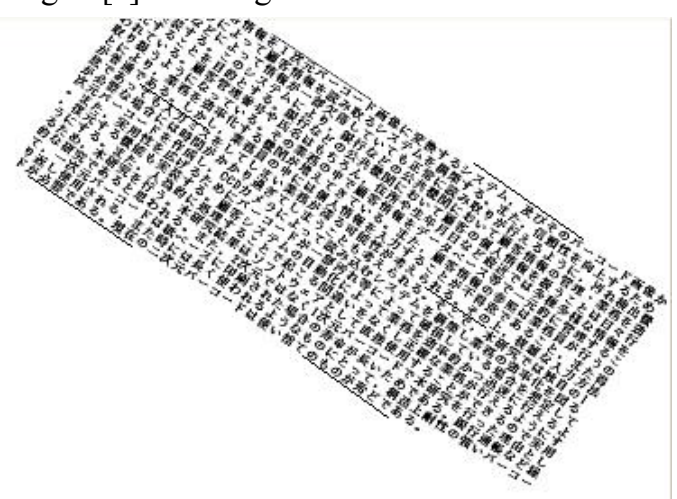

Fig.16[b] Incline gradient of document is $35^{\circ}$.

But error also happened as following. As shown in Fig. 17 the line has been drawn in the direction of the characters which have no relationship with each other. The gap between characters in English text is much bigger than that in Japanese text. In the case of Fig. 18, because in a certain angle, character does not exist by chance, error happens. But because the degree of error line is obviously different to correct one, correct degree can be judge easily with another 5 degrees.

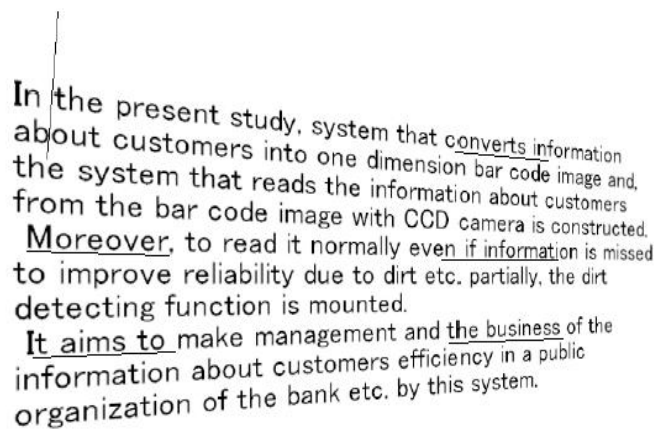

Fig.17 Error happens if gap between characters is big

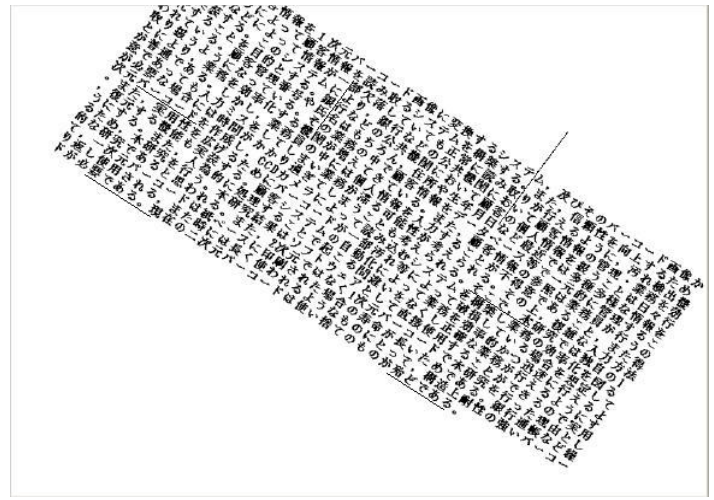

Fig.18 Error happens if characters missed by chance.

\section{Conclusions}

In this paper, we propose a method for getting the horizontal vanishing point of distorted camera document images with inclination of character strings. Our experimental results show that the proposed method can get the inclination gradient of the document images well and improve the method proposed in [27].In the next step, we will dewarp the image according the horizontal vanish point.

\section{Acknowledgment}

This work is supported by Prof.Serikawa, Prof. Lifeng Zhang, Huiming Lu. The author also gratefully acknowledges the helpful comments and suggestions of the reviewers, which have improved the presentation.

\section{References}

(1) J. Liang, D. Doermann, and H. Li. "Camera-based analysis of text and documents: a survey" International Journal on Document Analysis and Recognition, 7[2-3], 2005, pp. 84-104.

(2) F. Shafait, T. M. Breuel, "Document Image Dewarping Contest", In 2nd Int. Workshop on Camera-Based Document Analysis and Recognition, Curitiba, Brazil, 2007, pp. 181- 188.

(3) A.Yamashita, A.Kawarago, T.Kaneko, K.T.Miura, "Shape Reconstruction and Image Restoration for Non-Flat Surfaces of Documents with a Stereo Vision System", International Conference on Pattern Recognition, vol. 1, Cambridge, UK, 2004, pp. 482-485.

(4) M.S. Brown \& W.B. Seales, "Image Restoration of 
Arbitrarily Warped Documents", IEEE Trans. on Pattern Analysis and Machine Intelligence, 26[10], 2004, pp. 1295- 1306.

(5) C.L. Tan, L. Zhang, Z. Zhang \& T. Xia, "Restoring Warped Document Images through 3D Shape Modeling”, IEEE Trans. on Pattern Analysis and Machine Intelligence, 28[2], 2006, pp. 195-208.

(6) A. Ulges, C. H. Lampert, and T. Breuel, "Document capture using stereo vision" ACM Symposium on Document Engineering, Milwaukee, Wisconsin, USA, October 28-30, 2004, pp. 198-200.

(7) N. Gumerov, A. Zandifar, R. Duraiswarni, and L. S. Davis, "Structure of applicable surfaces from single views" European Conference on Computer Vision, Prague, 2004, pp.482-496.

(8) H. Cao, X. Ding and C. Liu, "A cylindrical surface model to rectify the bound document image" International Conference on Computer Vision, vol. 1, Nice, France, 2003, pp. 228.

(9) A. Masalovitch and L. Mestetskiy, "Usage of continuous skeletal image representation for document images dewarping" In 2nd Int. Workshop on Camera-Based Document Analysis and Recognition, Curitiba, Brazil, 2007, pp. 45-53.

(10) O. Lavaille, X. Molines, F. Angella and P. Baylou, "Active Contours Network to Straighten Distorted Text Lines" International Conference on Image Processing, Thessaloniki, Greece, 2001, pp. 1074-1077.

(11)Z. Zhang \& C. L. Tan, "Correcting document image warping based on regression of curved text lines" International Conference on Document Analysis and Recognition, Edinburgh, Scotland, 2003, pp. 589-593.

(12)H. Ezaki, S. Uchida, A. Asano \& H. Sakoe, "Dewarping of document image by global optimization" International Conference on Document Analysis and Recognition, Seoul, Korea, 2005, pp. 500-506.

(13)C. Wu \& G. Agam, "Document image De-warping for text/graphics recognition" Joint IAPR International Workshop on Structural, Syntactic, and Statistical Pattern Recognition, Windsor, Canada, 2002, pp. 348-357.

(14) A. Ulges, C.H. Lampert \& T.M. Breuel, "Document image dewarping using robust estimation of curled text lines" International Conference on Document Analysis and Recognition, Seoul, Korea, 2005, pp. 1001- 1005.

(15) Shijian Lu, Chew Lim Tan, "The Restoration of Camera Documents through Image Segmentation",
Workshop on Document Analysis Systems VIII, Nelson, New Zealand, 2006, pp. 484-495.

(16)B. Gatos, I. Pratikakis and I. Ntirogiannis, "Segmentation Based Recovery of Arbitrarily Warped Document Images" International Conference on Document Analysis and Recognition, Curitiba, Brazil, 2007, pp. 989- 993.

(17)J. Liang, D. DeMenthon, and D. Doermann, "Flattening curved documents in images" Conference on Computer Vision and Pattern Recognition, San Diego, USA, 2005, pp. 338-345.

(18) Y. C. Tsoi and M. S. Brown, "Geometric and shading correction for images of printed materials-A unified approach using boundary" Conference on Computer Vision and Pattern Recognition, Washington, DC, 2004, pp. 240- 246

(19)Z. Zhang \& C. L. Tan, "Warped image restoration with applications to digital libraries", International Conference on Document Analysis and Recognition, Seoul, Korea, 2005, pp. 192-196

(20) M. Wu, R. Li, B. Fu, W. Li and Z. Xu, "A Page Content Independent Book Dewarping Method to Handle 2D Images Captured by a Digital Camera", International Conference on Image Analysis and Recognition, Montreal, Canada, 2007, pp. 1242-1253.

(21)B. Gatos, I. Pratikakis \& S.J. Perantonis, "Adaptive Degraded Document Image Binarization", Pattern Recognition, 39, 2006, pp. 317-327.

(22)N. Stamatopoulos, B. Gatos, and A. Kesidis, "Automatic Borders Detection of Camera Document Images" In 2nd Int. Workshop on Camera-Based Document Analysis and Recognition, Curitiba, Brazil, 2007, pp. 71-78.

(23)U. V. Marti and H. Bunke, "Using a statistical language model to improve the performance of an HMM-based cursive handwriting recognition system", International journal of Pattern Recognition and Artificial intelligence, 15[1], 2001, pp. 65-90.

(24) http:/quito.informatik.uni-kl.de/dewarp/dewarp.php

(25) ABBYY FineReader OCR: http://finereader.abbyy.com/

(26) http://ocr.rossa.cc/digicamImage01p2.html

(27) http://pr.fujitsu.com/jp/news/2007/07/20-1.html 